\title{
A New Coefficient of Association between Two Attributes.
}

\author{
Kinosuke KIMURA
}

(The Impirial Fisheries Experimental Station, Tokyô)

Introduction. In statistics, the association between two attributes, that is the correlation between characters not quantitatively measurable has hitherto been studied by G. U. YULE ${ }^{(1)}$ and other investigators. They have introduced several coefficients for the measurement of the intensity of association. In the present paper, a new form is added to them.

When each individual of the observations, numbering $N$ in total, can

Table 1 .

\begin{tabular}{l|ll|l}
\hline Characters & $B$ & Non- $B$ & Total \\
\cline { 2 - 4 } $\begin{array}{c}A \\
\text { Non- } A\end{array}$ & $(A B)$ & $(A \beta)$ & $(A)$ \\
\hline Total & $(B B)$ & $(\alpha \beta)$ & $(\alpha)$ \\
\cline { 2 - 4 } & $(B)$ & $(\beta)$ & $N$ \\
\hline
\end{tabular}
be classified as being either $A$ or $\alpha$, i. e. as either possessing or not the attribute $A$, and at the same time, as being either $B$ or $\beta$, i.e. as either possessing or not the attribute $B$, the frequencies of actual coincidences between $A$ and $B, A$ and $\beta, \alpha$ and $B$ and $\alpha$ and $\beta$ are expressed as $(A B)$, $(A \beta),(\alpha B)$ and $(\alpha \beta)$ respectively, and they are set out in a "fourfold table" as shown in Table. 1 .

In case of complete independence of both attributes, the actual chance of coincidences, such as $(A B) \mid N$ is equall to the product of two probabilities, such as $(A) / N$ and $(B) \mid N$. Usually the difference $\delta_{A B}=(A B)-(A)(B) / N$ has been employed to calculate the coefficients of association ${ }^{(2)}$. For a fourfold table, we have $\delta_{A B}=\delta_{\alpha \beta}=-\delta_{A \beta}=-\delta_{a b}$ and this quantity is usually denoted by $\delta$.

The new coefficient of association. Now, the association between $A$ and $B$ is complete or the most intense when $\left|\delta_{A B}\right|$ assumes the gratest possible value. Expressing the greatest and the least possible value of $(A B)$ by $(A B)_{m i x}$ and $(A B)_{m i n}$ respectively, the new coefficient of association $K_{A B}$ between $A$ and $B$ is given by the following formulae:-

$$
K_{A B}=\frac{\delta_{A B}}{\left(\delta_{A B}\right)_{\max }}=\frac{(A B)-(A)(B) / N}{(A B)_{m+x}-(A)(B) \mid N} \text {, when } \delta_{A B}>0
$$

(1) Yule, G. U.: On the association of attributes in statistics. Phil. Trans. Roy. Soc A 194, 1900 , p. 257.

Journ. Roy. Stat. Soc., 75, 1912, p. 579 642.

(2) Recently late Prof. T. Terada used the ratio instead of the difference in his papers; TERadA, T.: Earthquakes and fisheries. Froc. Imp. Acad., 8, 1932, 83 86.

: Earthquake and fisheries II. Bull. Earthq. Res. Inst., 11, 1933, 714 716. 
and

$$
K_{A B}=\frac{\delta_{A B}}{\left(\delta_{A B}\right)_{m b n}}=\frac{(A B)-(A)(B) / N}{(A B)_{m i n}-(A)(B) / N} \text {, when } \quad \delta_{A B}<0 .
$$

Table 2

\begin{tabular}{c|cc|c} 
Characters & $B$ & Non-B & Total \\
\hline$A$ & $(B)$ & $(A)-(B)$ & $(A)$ \\
Non-A & 0 & $(\alpha)$ & $(\alpha)$ \\
\hline Total & $(B)$ & $(\beta)$ & $N$
\end{tabular}

Consider, for example, the cases where $\delta_{A B}>0$, and $(A)>(B)$. In such cases, $\delta_{A B}$ and $\delta_{\alpha, \beta}$ are maximum, while $\delta_{t, \beta}$ and $\delta_{a B}$ are minimum at the same time, when the association table takes the form as shown in Table 2. From Table 2 it follows that four coefficients, $K_{A B}, K_{A \beta}, K_{a, B}$ and $K_{a \beta}$, are

all the sane, because we have:-

$$
\begin{aligned}
K_{A B} & =\frac{(A B)-(A)(B) / N}{(B)-(A)(B) / N}=\frac{N \delta}{(B)\{N-(A)\}}=\frac{N \delta}{(\alpha)(B)}, \\
K_{A,} & =\frac{(A \beta)-(A)(\beta) / N}{(A)-(B)-(A)(\beta) / N}=\frac{-N}{(A)\{N-(\beta)\}-(B) N}=\frac{N \delta}{(\alpha)(B)}, \\
K_{a B} & =\frac{(\alpha B)-(\alpha)(B) / N}{-(\alpha)(B) / N}=\frac{-N \delta}{-(\alpha)(B)}=\frac{N \delta}{(\alpha)(B)}
\end{aligned}
$$

and

$$
K_{a \beta}=\frac{(\alpha \beta)-(\alpha)(\beta) / N}{(\alpha)-(\alpha)(\beta) / N}=\frac{N \delta}{(\alpha)\{N-(\beta)\}}=\frac{N \delta}{(\alpha)(B)}
$$

$K_{A \beta}$ or $K_{\alpha B}$ does not take a negative sign even if $\delta_{\Delta \beta}$ or $\delta_{\alpha B}$ is negative. When it is desired to distinguish positive and negative association, the denominators may be substituted by their absolute values.

Similar expressions for $K_{A B}$ as (3) can bo obtained in the cases other than $\delta_{A B}>0$ and $(A)>(B)$. But, the use of the formulae (1) and (2) is rather recommendable, where we have at once, from Table 1 , that $(A B)_{\max }$ is either $(A)$ or $(B)$ (whichever is the less) and $(A B)_{m l n}$ is either $(A)+(B)-N$ or 0 (whichever is the greater) $)^{(1)}$.

The new coefficient $K_{A B}$ fulfils, like other coefficients, the following conditions:- (i) $K_{A B}=0$, when $\delta_{A B}=0$; (ii) $K_{A B}=1$, when the association is

Table 3 .

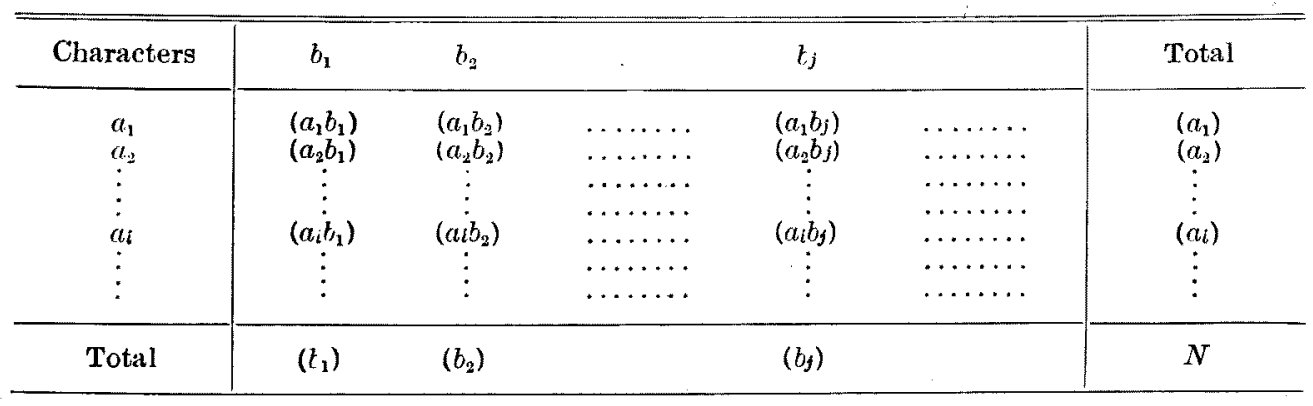

(1) Yule, G. U: An introduction to the thcory of statistic 3. 10 ed. London 1932, p. 29. 
most intense; and (iii) $K_{A B}$ increases and decreases continually, and in fact linearly, with $\delta_{A B}$, though $d K_{A B} / d \delta_{A B}$ is in general discontinuous at $\delta_{A B}=0$.

Manifold classification. When is the classification with regard to two attributes is manifold and the individual observation has either one of the characters $a_{1}, a_{3}, a_{3}$, etc. and at the same time either one of the second characters $b_{1}, b_{i}, b_{3}$, etc., the association table such as Table 3 can be transformed into a fourfold one with respect to $a_{l}$ and Non- $a_{\iota}$ and $b_{j}$ and Non- $b_{j}$, and $K_{a_{i} b}$ can be calculated as usual. But the result is obtainable directly from Table 3, because the formulae (1) and (2) can be write down by cising only $\left(a_{t}\right),\left(b_{j}\right),\left(a_{t} b_{j}\right)$ and $N$.

When one of the attributes is dichotomols as in Table 4, wo have always $K_{a_{1} b_{i}}=K_{a_{g^{b} b}}$ for all values of $i$.

Table 4.

\begin{tabular}{|c|c|c|c|c|c|c|}
\hline Characters & $b_{1}$ & $b_{2}$ & & $b_{l}$ & $\ldots \ldots$ & Total \\
\hline $\begin{array}{l}a_{1} \\
a_{2}\end{array}$ & $\begin{array}{l}\left(a_{1} b_{1}\right) \\
\left(a_{2} b_{1}\right)\end{array}$ & $\begin{array}{l}\left(a_{1} b_{2}\right) \\
\left(a_{2} b_{2}\right)\end{array}$ & $\ldots \ldots \ldots$ & $\begin{array}{l}\left(a_{1} b i\right) \\
\left(a_{2} b i\right)\end{array}$ & $\ldots \ldots \ldots$ & $\begin{array}{l}\left(a_{1}\right) \\
\left(a_{2}\right)\end{array}$ \\
\hline Total & $\left(l_{1}\right)$ & $\left(b_{2}\right)$ & $\ldots \ldots$ & $(b)$ & $\ldots \ldots \ldots$ & $N$ \\
\hline
\end{tabular}

In closing this paper, the author wishes to express his best thanks to: Mr. M. Okada for kind advices and also to Prof. T. Kameda for the permission to examine the literatures. 